\title{
Luminosity variations in several parallel auroral ares before auroral breakup
}

\author{
V. Safargaleev, W. Lyatsky, V. Tagirov \\ Polar Geophysical Institute, Apatity, 184200, Russia \\ Received: 18 March 1996 / Revised: 3 March 1997 / Accepted: 11 March 1997
}

\begin{abstract}
Variation of the luminosity in two parallel auroral arcs before auroral breakup has been studied by using digitised TV-data with high temporal and spatial resolution. The intervals when a new arc appears near already existing one were chosen for analysis. It is shown, for all cases, that the appearance of a new arc is accompanied by fading or disappearance of another arc. We have named these events out-of-phase events, OP. Another type of luminosity variation is characterised by almost simultaneous enhancement of intensity in the both arcs (in-phase event, IP). The characteristic time of IP events is $10-20 \mathrm{~s}$, whereas OP events last about one minute. Sometimes out-of-phase events begin as IP events. The possible mechanisms for OP and IP events are discussed.
\end{abstract}

\section{Introduction}

Research into the dynamics and morphology of auroras before auroral breakup may give information about possible reasons for substorm triggering. There are two well-known phenomena in auroras before the substorm onset: pseudobreakup events and auroral fading.

The term "pseudobreakup" was introduced by Akasofu (1964) for the description of local short-lived activation of auroral arcs. McPherron (1991) has noted that pseudobreakups are similar to the substorm expansion phase including a burst of Pi2 micropulsations and weak enhancement of westward electrojet. According to Koskinen et al. (1993) and Nakamura et al. (1994) the main difference between pseudobreakups and the substorm expansion onsets might be intensity and size of the disturbance source.

Correspondence to: V. Safargaleev

E-mailsafar@pgi-ksc.murmansk.su
Another prebreakup event is the auroral fading i.e. decrease in the discrete auroral arc luminosity a few minutes before an auroral breakup (Zaytseva et al., 1976; Pellinen and Heikkila, 1978). Kauristie et al. (1995) reported the ionospheric plasma depletion observed by EISCAT radar together with the arc fading just before the auroral breakup. Pudovkin et al. (1995) suggested that a specific $B_{z}$ distribution in the vicinity of the inner edge of the magnetotail plasma sheet hinders the particle precipitation from the Earthward-moving flux tube during the auroral fading.

Multiple auroral structures and their role in the substorm development have been investigated by many authors. The periodic (in longitude) arc structures at separations of more than $150 \mathrm{~km}$ were studied by Murphree et al. (1994). At a larger scale the observation of double auroral oval at the recovery phase of substorms was reported by Elphinstone et al. (1995b). In another paper by Elphinstone et al. (1995a), the possible role of periodic (in longitude) auroral structures in evolution of multiple onset substorms is discussed.

Relatively little attention was paid to correlation of the luminosity in the multiple auroral arc structures. In some papers for example, Persson et al. (1994) and Elphinstone et al. (1995b) the presence of several arcs in the sky was used to map the plasma sheet boundaries in the ionosphere and to define a region in the magnetotail where substorm onset takes place. Morse and Romick (1982) noted that the sky does not fade as a whole when more than one arc is observed, but some arcs dim while others brighten. Recently, Safargaleev et al. (1996) have reported an interesting case of observation of two auroral arcs a few minutes before an auroral breakup. They have found that activation of weak auroral arc leads to fading of more powerful equatorward arcs, and vice versa.

In the present study we extend this investigation, including some cases of simultaneous observation of two auroral arcs in the TV-camera field of view just before auroral breakups. 


\section{Method of investigation and description of events}

In our study we used the data of routine TV-camera registration of auroras at the Loparskaya observatory (corrected geomagnetic coordinates $64.70 \mathrm{~N}, 116.33 \mathrm{E}$; $\mathrm{MLT}=\mathrm{UT}+3 \mathrm{~h})$. The TV-camera was pointed toward zenith, the fish-eye lens gave a field of view of $170 \times 170^{\circ}$. We were interested in the intervals when multiple auroral arc structures (MAAS) were seen in the sky before a substorm onset. More than $60 \mathrm{~h}$ of TV data near the local midnight, obtained during the observation seasons of 1994-1995, were examined, but only three MAAS intervals of about $5 \mathrm{~min}$ in duration have been chosen for the further analysis. These data were digitised at $2 \mathrm{~s}$ intervals with spatial resolution of about $0.6 \times 0.6 \mathrm{~km}$ at an altitude of $110 \mathrm{~km}$ near the TVcamera zenith. The samples along the geographic meridian were used then to construct a keogram. Such keograms indicate the temporal behaviour of auroral luminosity as a function of latitude. The main features of each of the MAAS intervals is described next.

\subsection{January 30, 1995, 1727:15-1730:35 UT}

This interval is the most simple for analysis. The geomagnetic and aurora activity for the period is presented in Figs. 1, 2 where the MAAS interval is shown with either horizontal bars or black arrows. The development of the substorm is shown in Fig. 1a,b where variations of the horizontal component of the geomagnetic field at Lovozero observatory (corrected geomagnetic coordinates $64.09 \mathrm{~N}, 115.47 \mathrm{E} ; \mathrm{MLT}=$ $\mathrm{UT}+3 \mathrm{~h}$ ) are presented. The data from Kiruna and Sodankyla observatories are shown in Fig. 2, bottom right, the location of Kiruna, Sodankyla, Lovozero and Loparskaya may be seen in Fig. 2, bottom left.

As seen from the magnetograms in Figs. 1a and 2, the substorm expansive phase begins near 1725 UT as a strong negative excursion of the north component of the magnetic field in Lovozero, Sodankyla and Kiruna. At 1733 UT the next negative deviation takes place in Lovozero and Sodankyla. Both activations were accompanied by trains of Pi2 pulsations in Lovozero (see Fig. 1b). A few minutes before the second activation, during the relatively quiet period approximately $5 \mathrm{~min}$ in duration, the multiple auroral arc structure appears in the sky in Loparskaya.

Development of the auroras during the interval $1727-1730$ UT is shown in Fig. 2 (upper panel) as a sequence of all-sky images. The interval is characterised by appearance of a new arc approximately $70 \mathrm{~km}$ equatorward of a pre-existing arc. Appearance of the new arc was accompanied by fading of the poleward arc. Unfortunately, there is a data gap between 1730:351733:15 UT, and we cannot define the onset of the auroral breakup correctly. In accordance with the rapidrun magnetogram in Fig. 1b, its beginning may be defined close to 1733 UT. The auroras for this moment of time are shown in Fig. 2, bottom left (negative representation).
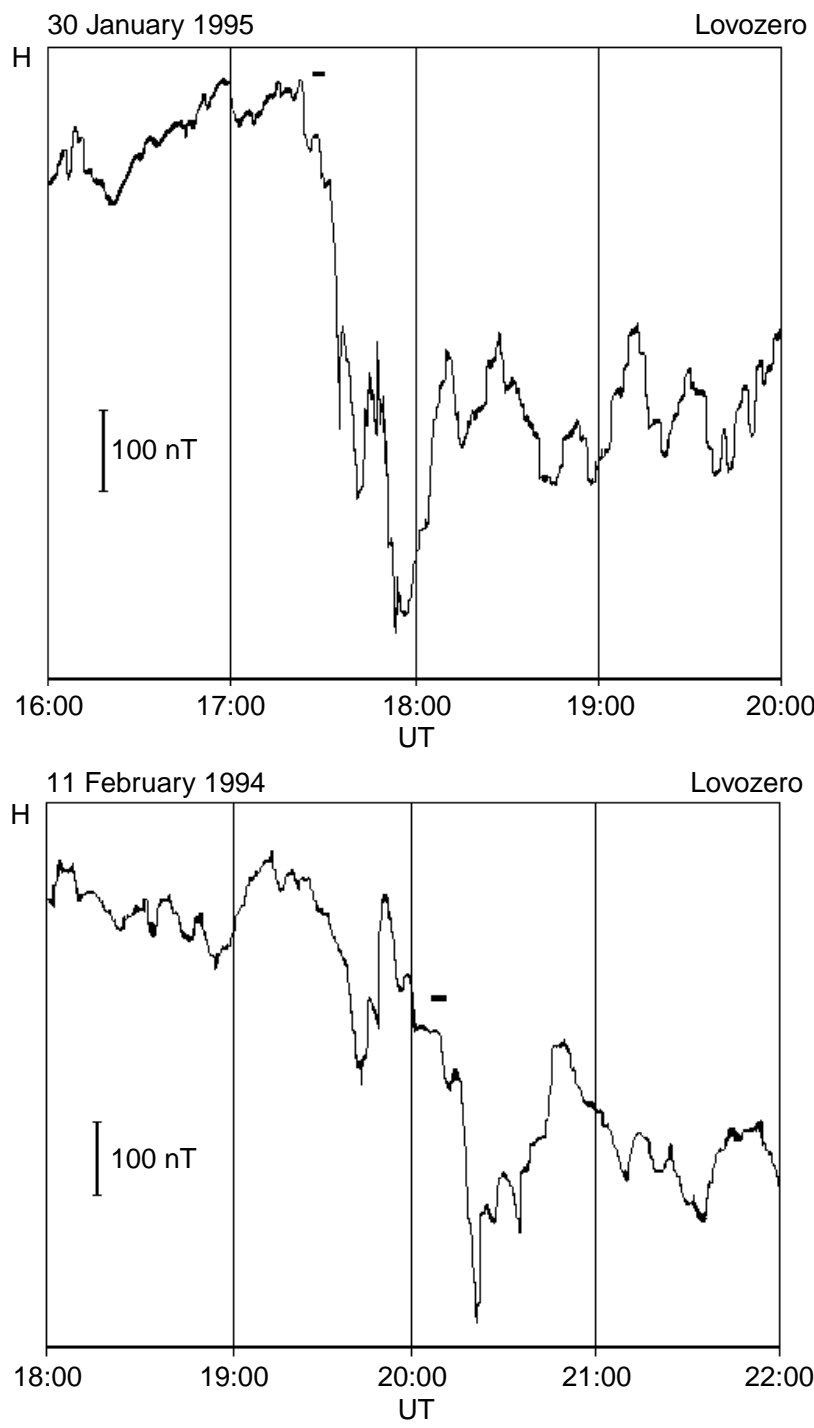

a

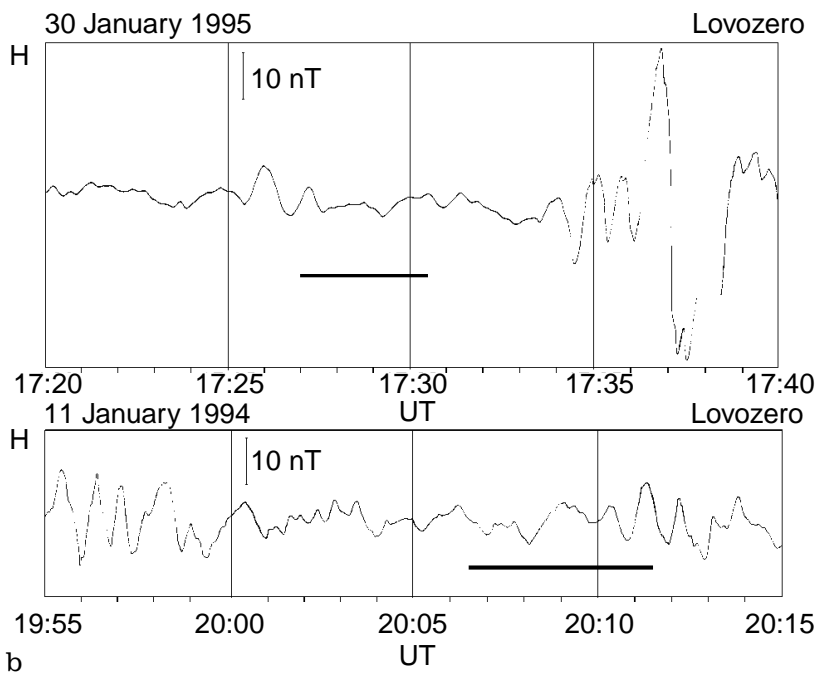

Fig. 1a,b. Variations of the H-component of geomagnetic field in Lovozero. a Standard magnetograms showing the level of geomagnetic activity. b Rapid-run magnetograms. The intervals of observation of multiple auroral arc structures (MAAS) are shown with horizontal bars 

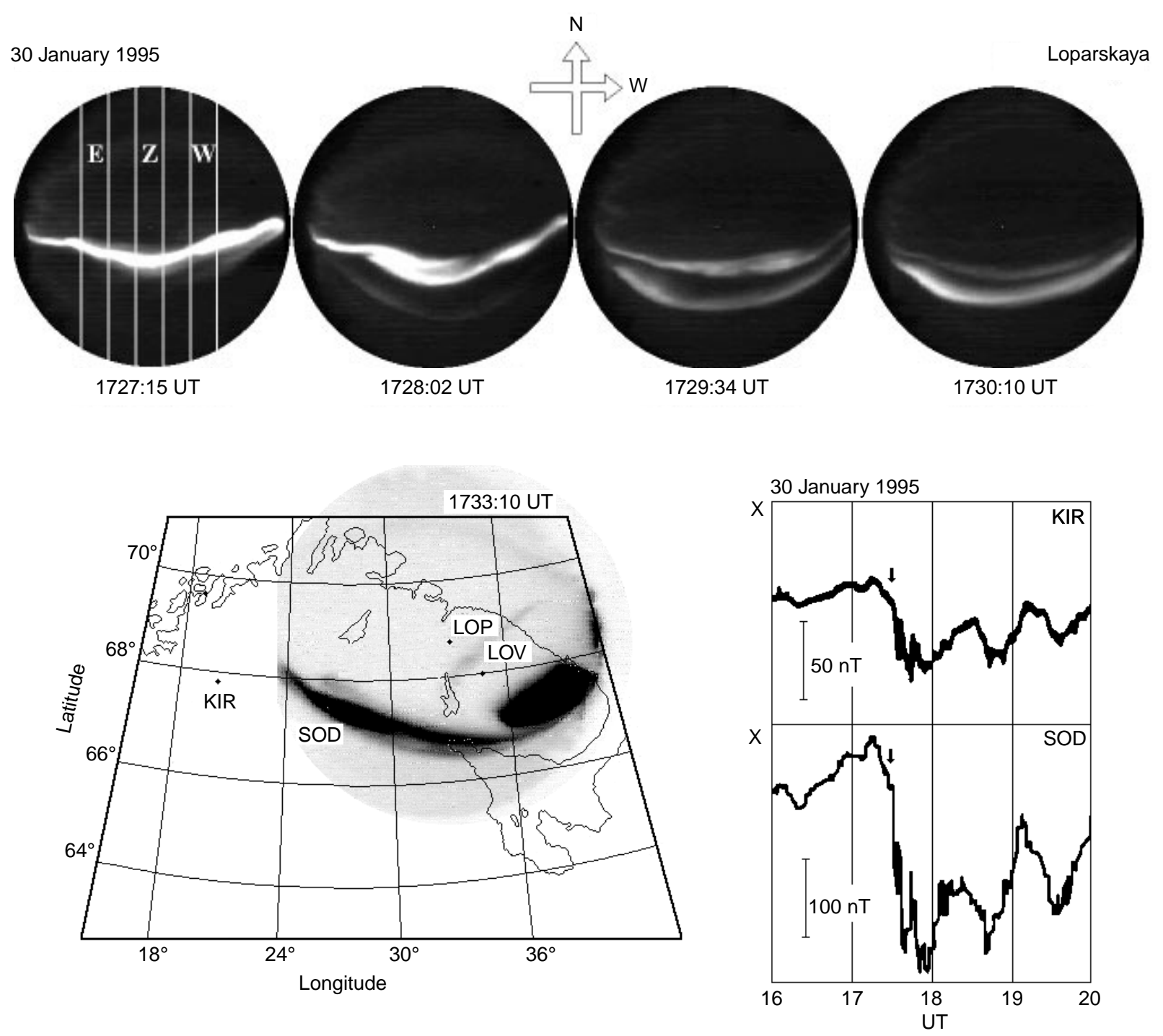

Fig. 2. Variations of the luminosity for two parallel auroral arcs as seen by TV-camera in Loparskaya; white lines on the first frame show three splits along which the (Z)enith, (E)astward and (W)estward profiles of the sky luminosity were obtained (top). Standard magnetograms showing the level of geomagnetic activity in Kiruna

In more detail, the variations of the mean value of the arc luminosity are presented in Fig. 3 (upper panel). Also shown are the keograms obtained along the (Z)enith, (E)astward and (W)estward profiles (lower panel). These profiles are indicated in Fig. 2 by white vertical lines on the first all-sky image. The keogram for the Z-profile indicates the temporal behaviour of sky luminosity along the geographic meridian of Loparskaya, $\mathrm{Z}^{\circ}$ means the zenith angle (positive to the north). The width of the Z-profile is about $25 \mathrm{~km}$ at an altitude of $110 \mathrm{~km}$ near the zenith. The keograms for the $\mathrm{W}$ - and E-profiles are presented to show the sky luminosity in the zenith vicinity. For convenience, we use the same $y$ axes as for the Z-profile. It is seen from these keograms that the arc fading as well as the arc brightness occurs almost simultaneously eastward and westward of the Zprofile and, hence, seems not to be caused by propagation of any small-scale irregularity along the arc.

and Sodankyla (bottom right), black arrows show MAAS interval. The co-location of TV-camera (Loparskaya) and magnetometers (Lovozero, Kiruna and Sodankyla); TV-camera field of view is shown at the altitude of $110 \mathrm{~km}$ (bottom left)

Two steps of auroral development can be seen during the period. At first, rapid fading of the poleward arc from 1727:30 to 1729:10 UT is accompanied by gradual increase in the luminosity of the equatorward arc. After 1729:10 UT, the luminosity of the equatorward arc increases more rapidly. We named such a type of arc activity the out-of-phase (OP) event. The beginning of each OP-event is indicated in Fig. 3 with a black arrow. There is another type of variation when the short-lived bursts of luminosity occurs in both arcs almost simultaneously (at $4 \mathrm{~s}$ accuracy) and may be seen both on Zprofile and $\mathrm{W}$ - and E-profiles. We named these bursts the in-phase (IP) events. Their beginnings are indicated in Fig. 3 with open arrows. Durations of the OP and IP events inside the interval are listed in Table 1 in the column entitled $\Delta \mathrm{T}$.

As seen in Fig. 3, the OP event at 1729:10 UT begins as an IP event. Similar luminosity behaviour in two arcs 


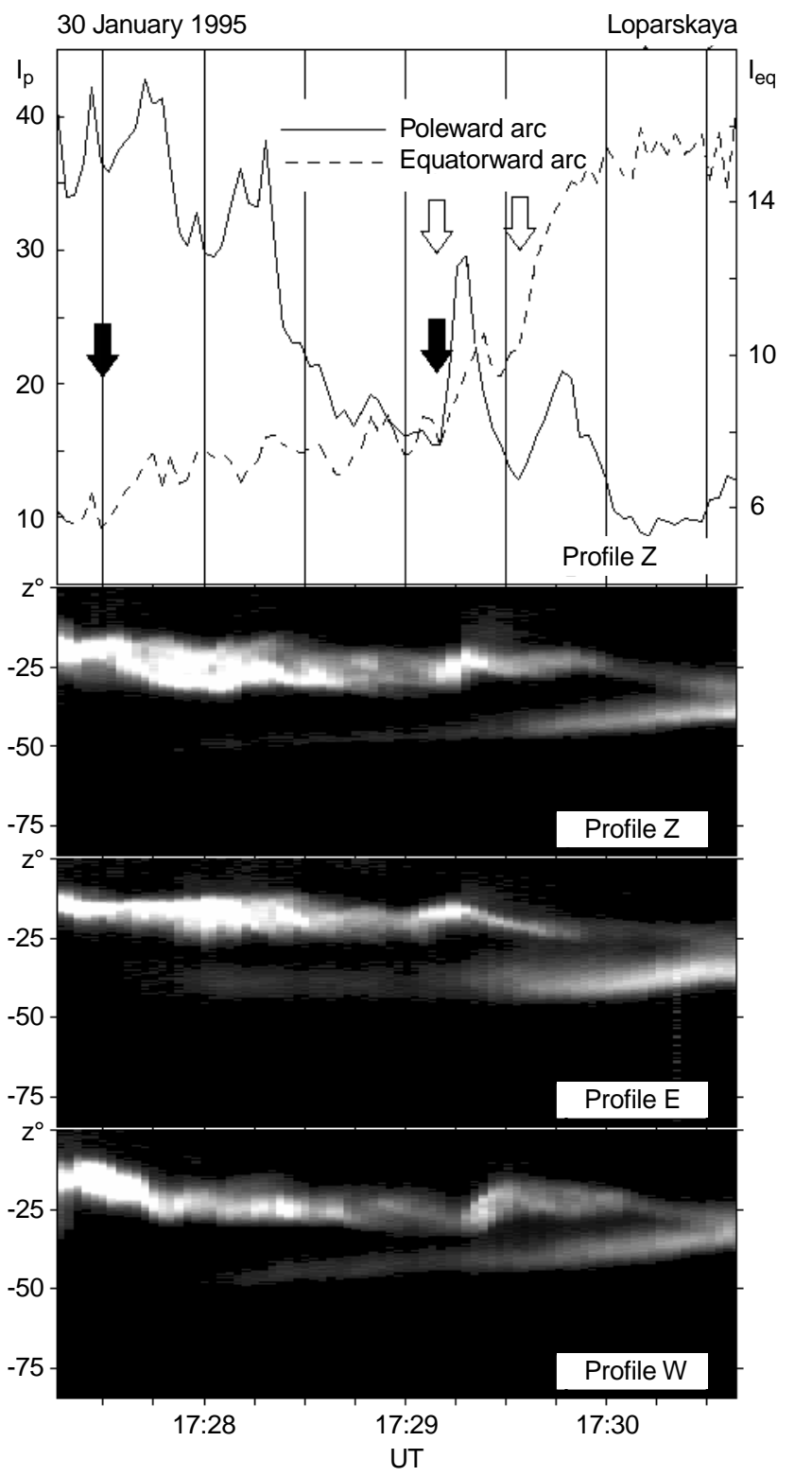

Fig. 3. Variations of the luminosity in two auroral arcs and grey-coded keograms for the MAAS interval on 30 January 1995. I means the mean value of the sky luminosity in the interval of zenith angles occupied by the arc (arbitrary units), subscripts " $p$ " and "eq" correspond to poleward and equatorward arc, respectively, $Z$ is the zenith angle. Black arrows show the beginning of OP events, open arrows show the beginning of IP events

(when the OP event begins as the IP one) may be seen also during the MAAS interval described earlier by Safargaleev et al. (1995).

\subsection{February 11, 1994, 2006:30-2011:30 UT}

The multiple auroral arc structure was observed during relatively a quiet interval a few minutes in duration between two consecutive substorm onsets (see magnetograms in Fig. 1a,b). Development of the auroras for this period is shown in Fig. 4. Auroral breakup begins at 2011 UT with formation of a westward travelling surge on the poleward arc.
Table 1. Parameters of OP and IP events. UT indicates the beginning of the event, $\Delta \mathrm{T}$ is the event duration

\begin{tabular}{llll}
\hline Date & UT & $\Delta \mathrm{T}(\mathrm{s})$ & Type \\
\hline $15.11 .93^{\mathrm{a}}$ & $1911: 00$ & 45 & OP \\
& $1912: 55$ & 60 & OP \\
& $1915: 30$ & 60 & OP \\
& $1912: 15$ & 15 & IP \\
& $1912: 30$ & 15 & IP \\
11.02 .94 & $1914: 55$ & 20 & IP \\
& $2007: 30$ & 60 & OP \\
& 2008.40 & 60 & OP \\
& $2010: 25$ & 50 & OP \\
30.01 .95 & $2007: 30$ & 20 & IP \\
& $2009: 30$ & 10 & IP \\
& 2010.25 & 10 & IP \\
& $1727: 30$ & 75 & OP \\
31.01 .95 & $1729: 10$ & 70 & OP \\
& $1729: 10$ & 15 & IP \\
& $1729: 35$ & 10 & IP \\
& $1852: 50$ & 45 & OP \\
& $1853: 40$ & 60 & OP \\
& $1847: 40$ & 10 & IP
\end{tabular}

${ }^{a}$ Data from the paper by Safargaleev et al. (1996)

There are two auroral arcs at separation of 50-70 km in the sky. Variations of luminosity in these arcs and keograms, are presented in Fig. 5. Three OP events may be seen in the interval. At first, the intensity of the poleward arc begins to decrease after 2007:30 UT when a new arc appears equatorward of the poleward one. One minute later, this new arc disappears while the poleward arc becomes more active again. Just before a break up, the poleward arc fades significantly. It is accompanied evidently by activation of the equatorward arc. These OP-events are indicated in Fig. 5 with black arrows. During the periods of activation of either the poleward or equatorward arc short-lived flares of luminosity are observed simultaneously in the both arcs (the IP-events, open arrows in Fig. 5). The times and durations of the OP and IP events are listed in Table 1.

Note that the activation just before the breakup begins as IP event (in both arcs simultaneously), but some seconds later the poleward arc disappears.

\subsection{January 31, 1995, 1852:30-1855:06 UT}

The geomagnetic and auroral activity for this interval is shown in Fig. 6. The first substorm in Lovozero begins at 1849 UT as a negative bay on the standard magnetogram (Fig. 6, upper panel) and as $\mathrm{Pi} 2$ enhancement on a rapid-run magnetogram (not shown). The WTS originated far away from the Loparskaya zenith. It spreads to the west, reaches Loparskaya and disappears. Some minutes later, the multiple auroral arc structure may be observed in Loparskaya. Both TV and all-sky camera observations had been stopped few seconds before the second substorm onset that occurred between 1855 and 1857 UT and was accompanied by $\mathrm{Pi} 2$ at 1855:25 UT.

The keogram for this interval is presented in Fig. 6 (lower panel). It is seen, that the appearance of a new arc 


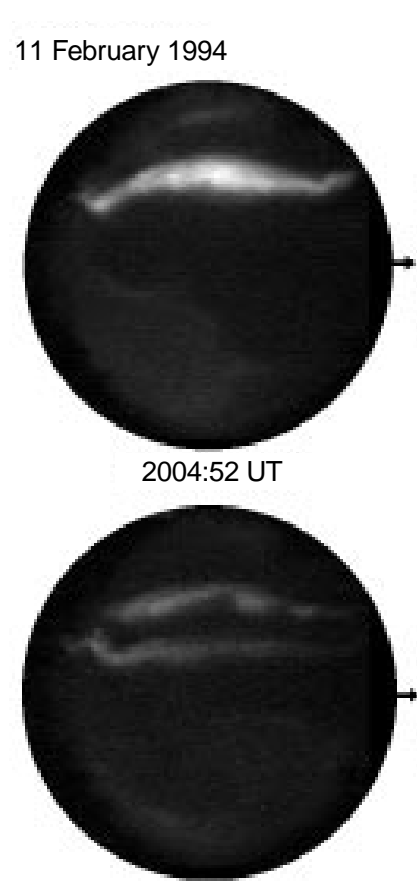

2010:28 UT

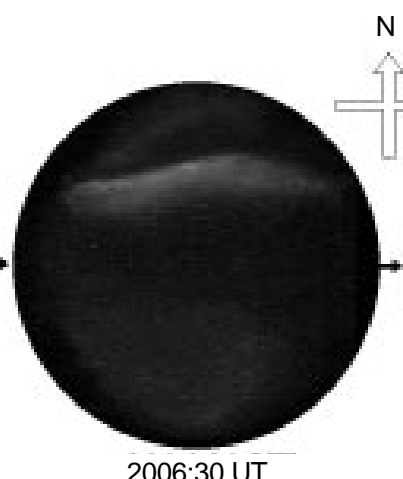

2006:30 UT

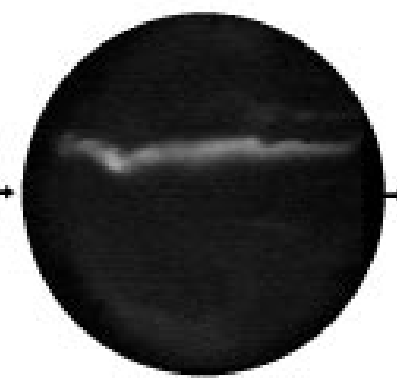

2010:50 UT

N
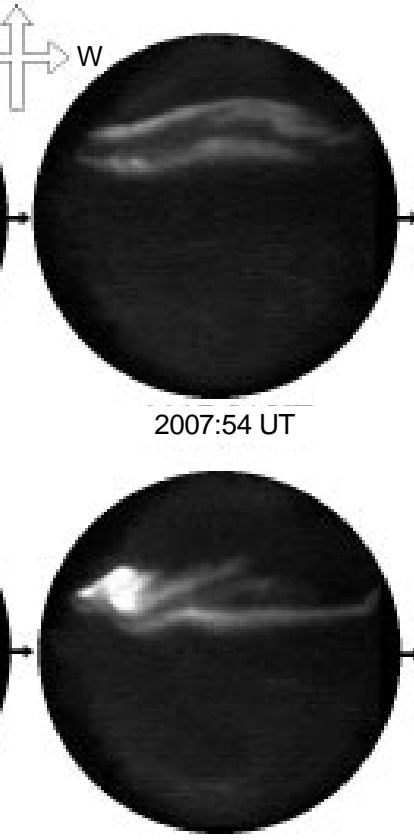

2011:02 UT

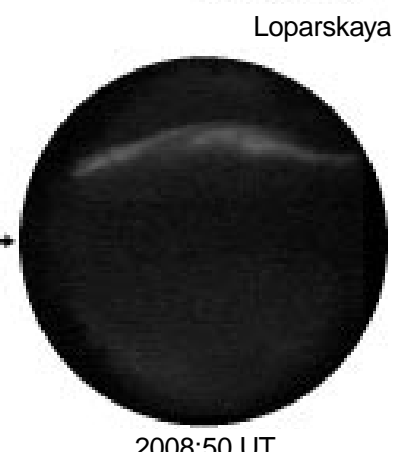

2008:50 UT

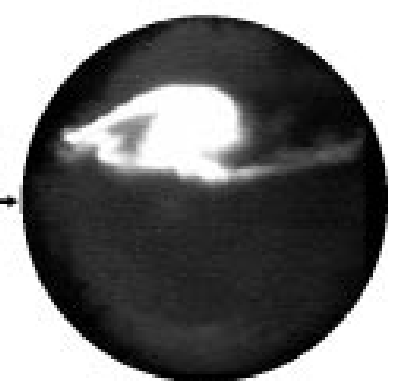

2011:18 UT

Fig. 4. Prebreakup activity during the MAAS interval on February 1994 showing the variations of the luminosity in several auroral arcs and the formation of the westward travelling surge

which begins few seconds before 1853 UT is accompanied by fading of the pre-existing arc located approximately $70 \mathrm{~km}$ to the north (the first OP event). At 1853:40 UT the poleward arc disappears almost completely, and the activity in the equatorward arc reaches its maximum. After that, the poleward arc becomes more active whereas the equatorward arc fades (the second OP events). These events are shown with black arrows in Fig. 6, the IP event is shown with open arrows, their times and durations are presented in Table 1 .

\section{Results and discussion}

In the present study we have described three intervals of prebreakup auroral activity when more than one auroral arc can be seen in the TV camera field-of-view.

We concentrated on the moments when a new arc appeared near the already existing one. We have found for all cases that the pre-existing arc then fades or disappears and named such events out-of-phase (OP) events. This result seems to be very important for understanding the mechanism for auroral arc formation. Next we discuss briefly the possible reasons for OPevents.

Most of the supposed mechanisms for arc formation have been examined by Borovsky (1993). We mention three that seem to be more quoted at present. In accordance with Atkinson (1992), formation of a new arc may be caused by magnetic field line reconnection in the distant magnetotail. The observed out-of-phase variations may show, in this case, that the reconnection onset at one point of the magnetotail plasma sheet hinders the reconnection development in another part of the magnetotail. On one hand, the reconnection leads to dipolarization of the magnetic field lines closing to the Earth. It creates unfavourable conditions for their merging and results in disappearance of the pre-existing equatorward arcs. On the other hand, the local reconnection in the magnetotail may produce a plasmoid in the magnetosphere equatorial plane (Hones et al., 1977). The plasmoid moves away from the Earth pushing out the magnetic field lines which also hinders the reconnection in the vicinity of the merging area and leads to fading of the pre-existing poleward arc.

In the "ionospheric-conductivity feedback instability" model, a small-scale patch of enhanced conductivity in the ionosphere plays a major role in forming of auroral arc (for instance, Sato and Holtzer, 1973). In accordance with Borowsky (1993), the width of the arc, $W_{\text {patch }}$, generated in this manner, depends on the magnitude of tangential component of the electric field, $W_{\text {patch }} \sim E_{E W}$. However, the simple electrostatic theory predicts that a narrow strip of enhanced conductivity should short-out this component. For this reason the active arc may "switch off" the arc in the vicinity.

In the model by Rothwell et al. (1991), an auroral arc is powered by the kinetic energy of Earthward-flowing plasmasheet plasma. As was mentioned, a new arc decreases the azimuthal component of the large-scale convection electric field in the ionosphere. This may reduces the Earthward flow of the plasma in the magnetosphere and, hence, the flow of energy which is necessary to support the existing arcs. 


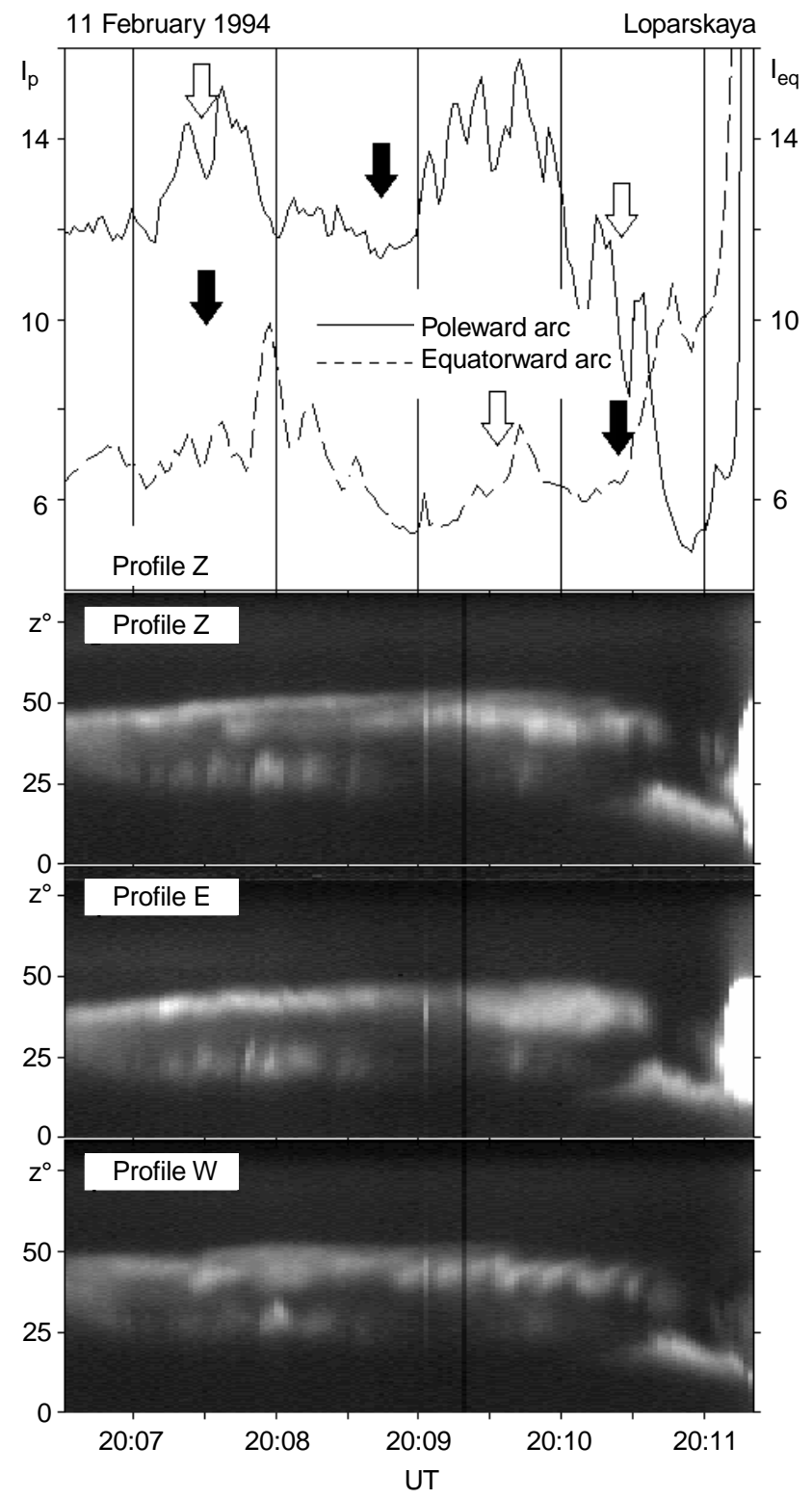

Fig. 5. The same as in Fig. 3 for the MAAS interval on 11 February 1994

We have presented three possible ways of explanation of out-of-phase variations. Another type of luminosity variations observed in our study seem to be more surprising phenomena. It includes the events of synchronous (defined with the accuracy of about $4 \mathrm{~s}$ ) brightening of the both arcs. We have used the term "in-phase (IP)" for description of those events. The duration of IP events is less than of OP events. This distinction between the events is seen clearly in Fig. 7, where the event number is plotted versus the event duration. It gives a characteristic time for IP events of 10-15 s whereas the OP events last about one minute.

The most interesting feature of IP events is the considerable synchronism of the observed variations. For this reason it is difficult to find any agent responsible for these events in the distant magnetotail. In fact, since the arcs are separated by $\sim 70 \mathrm{~km}$ in the
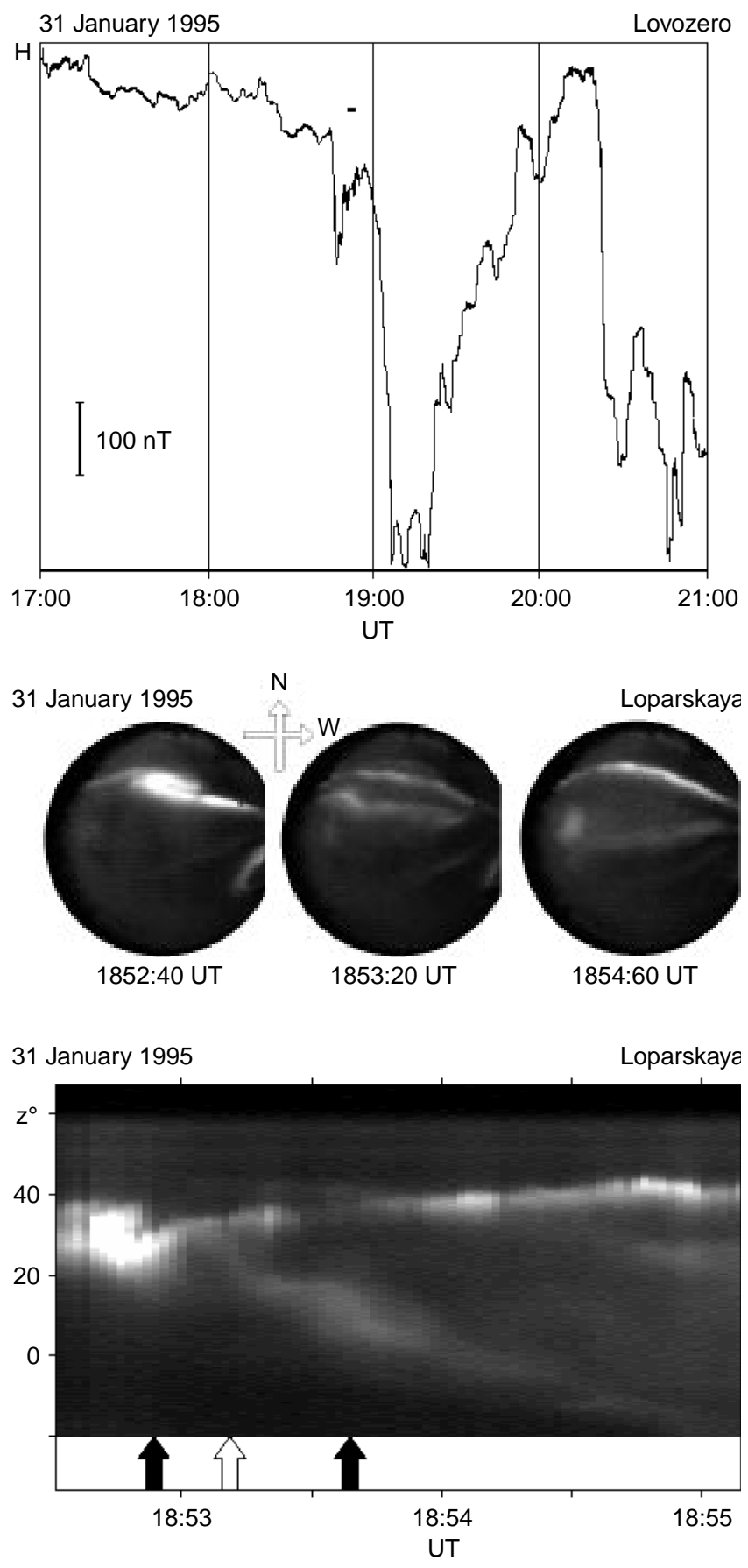

Fig. 6. Variations of the $H$-component of the magnetic field in Lovozero (upper panel) and development of the auroras (lower panel) for the MAAS interval on 30 January 1995. The horizontal line on the magnetogram shows the MAAS interval. A grey-coded keogram is obtained along the zenith profile. Black arrows mark the beginning of OP events, open arrow marks the beginning of IP event

ionosphere, the observed time delay (2-4 s) should give an extremely large value for the radial component of the velocity of the disturbance, $v_{r}$, in the equatorial plane of the magnetosphere. For instance, Tsyganenko's model for $K_{p}=4$ gives $v_{r} \sim 1500-3000 \mathrm{~km} \cdot \mathrm{s}^{-1}$ whereas the typical value of the Alfven velocity inside the magnetosphere is near $1000 \mathrm{~km} \cdot \mathrm{s}^{-1}$. It is more reasonable to 


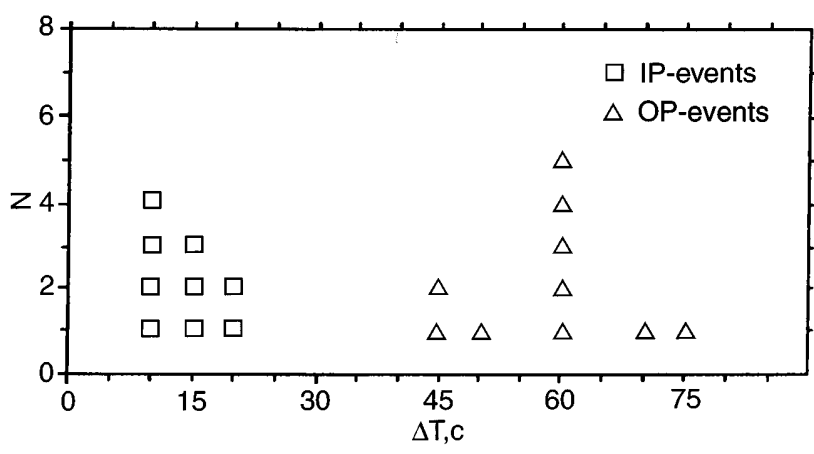

Fig. 7. Plot of the number of events versus the event duration. $I P$ signifies the event of simultaneous enhancement of the intensity in the arcs, $O P$ signifies the event of opposite behaviour of the arc intensity, $\Delta T$ is the duration of event

assume that particle acceleration during IP events takes place somewhere near the Earth, at low altitude. The anomalous resistivity could be a possible candidate for explanation of the IP events. A role for parallel electric field in the acceleration processes above discrete auroral forms was discussed, for instance, by Block and Falthammar (1990).

Another interesting feature of IP events is the possible connection with OP events. We have analysed four MAAS intervals and considered ten OP events. In three cases, the OP events just before the auroral breakup begin as IP events. This can be seen in Fig. 3 at 1729:10 UT, and in Fig. 5 at 2010:25 UT as well as in Fig. 5. of Safargaleev et al. (1996) near 1915 UT. As was mentioned the OP event may be caused by local reconnection in the magnetotail. From this point of view, the observed sequence of IP and OP events may mean triggering of the reconnection by some agent propagating from the dayside magnetosphere to the magnetotail. Additional investigation of this problem is necessary because it seems to be important to understand the mechanism for substorm triggering.

Unfortunately, the low quality of the magnetic data does not allow us to connect either OP or IP events with any change in the magnetic field variations, especially, in $\mathrm{Pi} 2$ and $\mathrm{PiB}$ pulsations. This extremely interesting question remains beyond the scope of this study.

A case concerning the observation of out-of-phase pulsating "auroral bistructure" (i.e. two discrete auroral arcs at a separation of a few tens of kilometers) during the substorm expansion phase has been reported by Kornilova et al. (1982). The period of these pulsations was found to be about a few seconds. Pulsating anomalous resistivity was discussed as a possible reason for that event. The oscillating auroral phenomena associated directly with substorm expansion and its possible connection with waves in the plasma sheet was discussed by Liu et al. (1995). The period of observed oscillations was $\sim 10 \mathrm{~min}$.

In connection with our study, it is interesting also to mention the results by Murphree et al. (1994). They investigated spatially periodic auroras and pointed out that no periodic structures were found at a separation of less than $100 \mathrm{~km}$. It is in good agreement with our results. In accordance with our observations, two active arcs at separation less than $100 \mathrm{~km}$ could not co-exist over a long time.

\section{Conclusions}

1. Luminosity variations in two parallel auroral arcs before auroral breakup have been studied. It is shown that events of short-lived activation at least in one of the arcs may be separated into two groups. The events of the first group are characterised by opposite behaviour of the arc intensity when the appearance of a new arc is accompanied by fading or disappearance of the preexisting arc (the out-of-phase events, OP). The events of another group are characterised by simultaneous (at $4 \mathrm{~s}$ accuracy) enhancement in intensity in all arcs (the inphase events, IP).

2. The characteristic duration of the IP events is $10-20 \mathrm{~s}$, whereas the OP events last about one minute. Often the events begin as an IP event, but then transform into an OP event.

3. A possible explanation of the OP events is discussed in frames of different theories for auroral arc formation. In particular, the observed out-of-phase variations of the arc luminosity may mean that the beginning of the reconnection in one part of the magnetotail plasma sheet hinders the reconnection development in the vicinity of the merging area. To satisfy the observed simultaneity of short-lived luminosity enhancement during the IP events, the region of the auroral particle acceleration seems to be located at low altitudes.

Acknowledgements. This material was based upon research supported by the Russian Foundation for Fundamental Researches (Grant 05-95-14496). Authors thank V. N. Kriviliov (Polar Geophysical Institute) for his help in data development. Kiruna and Sodankyla magnetometer data are taken from Kiruna Geophysical Data, data summary 95/1-3, January-March 1995, Kiruna (ed. Sh. Kirkwood) and Geomagnetic, ionospheric and auroral data from Finland, monthly bulletin, January 1995, Sodankyla.

Topical Editor D. Alcaydé thanks T. Bösinger and another referee for their help in evaluating this paper.

\section{References}

Akasofu, S. -I., The development of auroral substorm, Planet. Space Sci., 12, 273-388, 1964.

Atkinson, G., Mechanism by which merging at $\mathrm{X}$ line causes discrete auroral arcs, J. Geophys. Res., 97, 1337-1342, 1992.

Block, L. R., and C.-G. Falthammar, The role of magnetic-fieldaligned electric fields in auroral acceleration. J. Geophys. Res., 95, 5877-5888, 1990.

Borovsky, J. E., Auroral arc thicknesses as predicted by various theories, J. Geophys. Res., 98, 6101-6138, 1993.

Elphinstone, R. D., D. J. Hearn, L. L. Cogger, et al., Observations in the vicinity of substorm onset: implications for the substorm process. J. Geophys. Res., 100, 7937-7969, 1995a.

Elphinstone, R. D., J. S. Murphree, D. J. Hearn, et al., The double oval UV auroral distribution, 1, implication for the mapping of auroral arcs, J. Geophys. Res., 100, 12075-12093, 1995b.

Hones, E. W., Jr., Substorm processes in the magnetotail: comments on "On hot tenuous plasmas, fairballs, and boundary 
layers in the earth's magnetotail" by L.A. Frank, et al., J. Geophys. Res., 82, 5633-5640, 1977.

Kauriste, K., T. I. Pulkkinen, R. J. Pellinen, P. Janhunen, A. Huuskonen, A. Viljanen, H. J. Opgenoorth, W. J. Heikkila, and D. N. Baker, Analysis of the substorm trigger phase using multiple ground-based instrumentation, Geophys. Res. Lett., 22, 2065-2068, 1995.

Kornilova, T. A., T. N. Kolosova, M. L. Pudovkin, Pulsating auroral bistructures and conductivity of the magnetospheric plasma, Auroras, 30, 58-62, 1982 (in Russian).

Koskinen, H. E. J., R. E. Lopez, R. J. Pellinen, T. I. Pulkkinen, D. N. Baker, and T. Bosinger, Pseudobreakup and substorm growth phase in the ionosphere and magnetosphere, J. Geophys. Res., 98, 5801-5813, 1993.

Liu, W. W., B. -L. Xu, J. S. Samson, and G. Rostoker, Theory and observation of auroral substorms: a magnetohydrodynamic approach, J. Goephys. Res., 100, 79-95, 1995.

McPherron, R. L., Physical processes producing magnetospheric substorms: and magnetic storms, in Geomagnetism, vol. 4, Ed J.A. Jacobs, p.593, Academic, San Diego, Calif., 1991.

Morse, T. H., and G. J. Romick, The fluctuation and fading of auroral arcs preceding auroral substorm onsets, Geophys. Res. Lett., 9, 1065-1068, 1982.

Murphree, J. S., M. L. Johnson, L. L. Cogger and D. J. Hearn. Freja UV imager observations of spatially periodic auroral distortions, Geophys. Res. Lett., 21, 1887-1890, 1994.
Nakamura, R., D. N. Baker, T. Yamamoto, R. D. Belian, E. A. Bering III, J. R. Benbrook, and J. R. Theall, Particle and field signatures during pseudobreakup and major expansion onset, $J$. Geophys. Res., 90, 207-221, 1994.

Pellinen, R. J., and W. J. Heikkila, Observation of aurora fading before breakup, J. Geophys. Res., 83, 4207-4218, 1978.

Persson, M. A. L., H. J. Opgenoorth, T.E. Pulkkinen, et al., NearEarth substorm onset: a coordinated study, Geophys. Res. Lett., 21, 1875-1878, 1994.

Pudovkin, M. I., S. A. Zaytseva, T. A. Kornilova, R. I. Pellinen, Dynamics of the auroras near the equatorward edge of auroral oval, Geomagn. Aeron., 35, 47-52, 1995 (in Russian).

Rothwell, P. L., M. B. Silevitch, L. P. Block, and C. -G. Falthammar, Prebreakup arcs: a comparison between theory and experiment, J. Geophys. Res., 96, 13967-13979, 1991.

Safargaleev, V., T. Turunen, W. Lyatsky, J. Manninen, and A. Kozlovsky, Imaging and EISCAT radar measurements of an auroral prebreakup event, Annales Geophysicae, 14, 11701176, 1996.

Sato, T., and T. E. Holtzer, Quiet auroral arcs and electrodynamic coupling between the ionosphere and the magnetosphere, 1, J. Geophys. Res., 78, 7314-7322, 1973.

Zaytseva, S. A., B. M. Kuznetsov, M. I. Pudovkin., Dynamics of the auroras and currents during substorm, Geomagn. Issled., 18, 7378, 1976 (in Russian). 